Research Article

\title{
Noether Symmetry Method for Hamiltonian Mechanics Involving Generalized Operators
}

\author{
Chuan-Jing Song $(\mathbb{D}$ and Yao Cheng $(\mathbb{D})$ \\ School of Mathematical Sciences, Suzhou University of Science and Technology, Suzhou 215009, China \\ Correspondence should be addressed to Chuan-Jing Song; songchuanjingsun@126.com
}

Received 22 August 2021; Accepted 13 September 2021; Published 29 September 2021

Academic Editor: Ivan Giorgio

Copyright (c) 2021 Chuan-Jing Song and Yao Cheng. This is an open access article distributed under the Creative Commons Attribution License, which permits unrestricted use, distribution, and reproduction in any medium, provided the original work is properly cited.

Based on the generalized operators, Hamilton equation, Noether symmetry, and perturbation to Noether symmetry are studied. The main contents are divided into four parts, and every part includes two generalized operators. Firstly, Hamilton equations within generalized operators are established. Secondly, the Noether symmetry method and conserved quantity are studied. Thirdly, perturbation to the Noether symmetry and adiabatic invariant are presented. And finally, two applications are presented to illustrate the methods and results.

\section{Introduction}

With the development of discipline and the progress of technology, the dynamics of the constrained mechanical systems was put forward, so Analytical Mechanics appears. Lagrange is the founder of Analytical Mechanics. Lagrange further studied the motion of the constrained particles after d'Alembert. The Lagrange equation and the d'Alembert-Lagrange principle are the core of Lagrangian mechanics.

Hamilton developed Analytical Mechanics. In his two long papers, "On a General Method in Dynamics" (1834) and "Second Essay on a General Method in Dynamics" (1835), he proposed an integral variational principle and a dynamic equation with generalized coordinate and generalized momentum as independent variables. This principle is called the Hamilton principle. The dynamic equation given by Hamilton is called the canonical equation. The Hamilton principle and the Hamilton canonical equation are the core of Hamiltonian mechanics.

Hamilton's principle is highly general, which can represent the motion law of a holonomic and conservative system by only one functional extreme value. The principle is not only simple and beautiful in form but also rich and profound in connotation. It can be applied to mechanics, optics, electromagnetism, and other fields and can also be applied to approximate calculation [1, 2]. In Ref. [3], the Hamilton principle is applied to the dynamics of the flexible multibody and rigid flexible coupling systems. Hamilton's principle is extended to the holonomic nonconservative system [4], the high-order system [5], and the nonholonomic system [4]. In addition, the Pfaff-Birkhoff principle [6,7], generalized Pfaff-Birkhoff principle [8], and Vujanović's variational principle of nonconservative system [9] are also the generalization of Hamilton's principle.

The Hamilton equation is also of great significance. Firstly, the canonical equation is simpler in form and more symmetrical in structure than the Lagrange equation. It is more convenient for general discussion when solving many complex mechanical problems, such as celestial mechanics and vibration theory. Secondly, the new concepts related to the canonical equations, such as the canonical variables, have many applications in mechanics and physics, such as statistical physics and quantum mechanics. Thirdly, a complete set of integration methods are established for the canonical equations, such as the Poisson theorem, Jacobi method, canonical transformation, and integral invariants [4]. Fourthly, geometric mechanics has been developed due to the symplectic structure of the canonical equations. Besides, Hamiltonian mechanics has also contributed to the formation and the development of the generalized 
Hamiltonian mechanics [10] and the Birkhoffian mechanics [11]. Finally, considering the perturbation of the Hamilton equation, the KAM theorem appears. The KAM theorem became the beginning of the chaos theory [4].

In 1918, the famous paper "Invariante Variations Probleme" by German mathematician Noether revealed the relationship between the conserved quantity of the mechanics system and its internal dynamical symmetry [12]. Reference [13] points out that the application of the mechanics variational principle and its physical significance are based on two theorems: the Hilbert independence theorem and the Noether theorem. The first theorem gives the mathematical argument of the variational principle, and the second theorem reveals its physical significance.

The Noether symmetry method is one of the modern integration methods of the Hamiltonian mechanics. For the Hamiltonian system, Noether symmetry is the invariance of the Hamilton action under the infinitesimal transformations. The Noether symmetry method points out that if the infinitesimal generators and the gauge function satisfy the Noether identity, then the conserved quantity of the system can be found [14-20]. The advantage of the Noether theory is that if there is a Noether symmetry, a corresponding conserved quantity can be found and vice versa. The Noether theory can also be used to solve general ordinary differential equations as long as they are expressed as equations of mechanics systems [21].

Recent developments in the fields of science, engineering, economics, bioengineering, and applied mathematics have demonstrated that many phenomena in nature are modelled more accurately using fractional derivatives [22, 23]. Fractional Hamiltonian mechanics [24-32] and Noether theorems for them have also been established and investigated [33-36]. The fractional operators mainly referred to the left and right fractional Riemann-Liouville integrals, the left and right fractional Riemann-Liouville differential operators, the left and right fractional Caputo differential operators, the Riesz-Riemann-Liouville differential operator, and the Riesz-Caputo differential operator. However, Agrawal [37] pointed out that the fractional power kernel need not be the only kernel to describe the phenomena of the nature. He defined three new operators which in special cases reduce to the fractional operators listed above. Then, the entire theories of the Hamiltonian mechanics and the corresponding Noether theorem can be redeveloped. And in such a case, the theories of the integer order and the fractional order Hamiltonian mechanics and other results resulted from them would be special cases of the more general new operators.

The structure of this paper is organized as follows. Section 2 lists the definitions and properties of the generalized operators briefly. Fractional calculus of variations for the Hamiltonian system within generalized operators is studied in Section 3. Based on the generalized operators, Noether symmetry, and conserved quantities, perturbation to Noether symmetry and adiabatic invariants are investigated in Section 4 and Section 5, respectively. Section 6 presents two applications to illustrate the methods and results obtained in this paper. A conclusion is given in Section 7.

\section{Preliminaries}

The definitions and properties of the generalized operators $K, A$, and $B$ were studied in detail by Agrawal [37]. Here, we only list their definitions and integration by part formulae.

The operators $K, A$, and $B$ have the forms

$$
\begin{gathered}
K_{M}^{\alpha} f(t)=m \int_{a}^{t} \kappa_{\alpha}(t, \tau) f(\tau) \mathrm{d} \tau+\omega \int_{t}^{b} \kappa_{\alpha}(\tau, t) f(\tau) \mathrm{d} \tau, \quad \alpha>0, \\
A_{M}^{\alpha} f(t)=D^{n} K_{M}^{n-\alpha} f(t), \quad n-1<\alpha<n \\
B_{M}^{\alpha} f(t)=K_{M}^{n-\alpha} D^{n} f(t), \quad n-1<\alpha<n
\end{gathered}
$$

where $f(t)$ is continuous and integrable, $a<t<b, M=<a$, $t, b, m, \omega>$ is a parameter set, $m$ and $\omega$ are two real numbers, $\kappa_{\alpha}(t, \tau)$ is a kernel which may depend on a parameter $\alpha$, and $n$ is an integer.

Remark 1. Let $\kappa_{\alpha}(t, \tau)=(t-\tau)^{\alpha-1} / \Gamma(\alpha)$, then different results will be obtained under different conditions. For example, when $M=M_{1}=\langle a, t, b, 1,0\rangle$, we have

$$
\begin{aligned}
A_{M}^{\alpha} f(t) & =D^{n} K_{M}^{n-\alpha} f(t)=\frac{1}{\Gamma(n-\alpha)}\left(\frac{\mathrm{d}}{\mathrm{d} t}\right)^{n} \int_{a}^{t}(t-\tau)^{n-\alpha-1} f(\tau) \mathrm{d} \tau \\
& ={ }_{a}^{R L} D_{t}^{\alpha} f(t), \\
B_{M}^{\alpha} f(t) & =K_{M}^{n-\alpha} D^{n} f(t)=\frac{1}{\Gamma(n-\alpha)} \int_{a}^{t}(t-\tau)^{n-\alpha-1}\left(\frac{\mathrm{d}}{\mathrm{d} \tau}\right)^{n} f(\tau) \mathrm{d} \tau \\
& ={ }_{a}^{C} D_{t}^{\alpha} f(t),
\end{aligned}
$$

i.e., the operator $A$ and the operator $B$ reduce to the left Riemann-Liouville fractional operator and the left Caputo fractional operator, respectively. When $M=M_{2}=<a, t, b, 0$, $1>$, we have

$$
\begin{aligned}
A_{M}^{\alpha} f(t) & =D^{n} K_{M}^{n-\alpha} f(t)=\frac{1}{\Gamma(n-\alpha)}\left(\frac{\mathrm{d}}{\mathrm{d} t}\right)^{n} \int_{t}^{b}(\tau-t)^{n-\alpha-1} f(\tau) \mathrm{d} \tau \\
& =(-1)_{t}^{n R L} D_{b}^{\alpha} f(t), \\
B_{M}^{\alpha} f(t) & =K_{M}^{n-\alpha} D^{n} f(t)=\frac{1}{\Gamma(n-\alpha)} \int_{t}^{b}(\tau-t)^{n-\alpha-1}\left(\frac{\mathrm{d}}{\mathrm{d} \tau}\right)^{n} f(\tau) \mathrm{d} \tau \\
& =(-1)_{t}^{n C} D_{b}^{\alpha} f(t),
\end{aligned}
$$

i.e., the operator $A$ and the operator $B$ reduce to the right Riemann-Liouville fractional operator and the right Caputo fractional operator, respectively. When $M=M_{3}=<a, t, b, 1$ / $2,1 / 2>$, we have 


$$
\begin{aligned}
A_{M}^{\alpha} f(t) & =D^{n} K_{M}^{n-\alpha} f(t)=\frac{1}{2 \Gamma(n-\alpha)}\left(\frac{\mathrm{d}}{\mathrm{d} t}\right)^{n} \int_{a}^{b}|t-\tau|^{n-\alpha-1} f(\tau) \mathrm{d} \tau \\
& ={ }_{a}^{R} D_{b}^{\alpha} f(t), \\
B_{M}^{\alpha} f(t) & =K_{M}^{n-\alpha} D^{n} f(t)=\frac{1}{2 \Gamma(n-\alpha)} \int_{a}^{b}|t-\tau|^{n-\alpha-1}\left(\frac{\mathrm{d}}{\mathrm{d} \tau}\right)^{n} f(\tau) \mathrm{d} \tau \\
& ={ }_{a}^{R C} D_{b}^{\alpha} f(t),
\end{aligned}
$$

i.e., the operator $A$ and the operator $B$ reduce to the RieszRiemann-Liouville fractional operator and the Riesz-Caputo fractional operator, respectively.

The generalized operators $K, A$, and $B$ satisfy the following integration by part formulae:

$$
\begin{gathered}
\int_{a}^{b} g(t) K_{M}^{\alpha} f(t) \mathrm{d} t=\int_{a}^{b} f(t) K_{M^{*}}^{\alpha} g(t) \mathrm{d} t \\
\int_{a}^{b} g(t) A_{M}^{\alpha} f(t) \mathrm{d} t=(-1)^{n} \int_{a}^{b} f(t) B_{M^{*}}^{\alpha} g(t) \mathrm{d} t \\
+\left.\sum_{j=0}^{n-1}(-D)^{n-1-j} g(t) A_{M}^{\alpha+j-n} f(t)\right|_{t=a} ^{t=b} \\
\int_{a}^{b} g(t) B_{M}^{\alpha} f(t) \mathrm{d} t= \\
+\sum_{j=0}^{n-1}(-1)^{n} \int_{a}^{b} f(t) A_{M^{*}}^{\alpha} g(t) \mathrm{d} t
\end{gathered}
$$

where $M^{*}=\langle a, t, b, \omega, m\rangle, n-1<\alpha<n$, and $n$ is an integer.

In this text, we set $n=1$, so $0<\alpha<1$. The variational problems within generalized operators $A$ and $B$ are to be studied first.

\section{Hamilton Equations within Generalized Operators}

3.1. Hamilton Equation within Generalized Operator A. Let $L_{A}=L_{A}\left(t, q_{A}, A_{M}^{\alpha} q_{A}\right)$ be the Lagrangian within generalized operator $A, q_{A}=\left(q_{A 1}, q_{A 2}, \cdots, q_{\mathrm{An}}\right)$, and $A_{M}^{\alpha} q_{A}=\left(A_{M}^{\alpha} q_{A 1}, A_{M}^{\alpha}\right.$ $\left.q_{A 2}, \cdots, A_{M}^{\alpha} q_{\mathrm{An}}\right)$, then the generalized momentum $p_{A}=\left(p_{A 1}\right.$, $\left.p_{A 2}, \cdots, p_{\text {An }}\right)$ is defined as $p_{A i}=\partial L_{A} / \partial A_{M}^{\alpha} q_{A i}, i=1,2, \cdots n$, and the Hamiltonian $H_{A}=H_{A}\left(t, q_{A}, p_{A}\right)$ can be expressed as $H_{A}=p_{A i} \cdot A_{M}^{\alpha} q_{A i}-L_{A}$. Therefore, the Hamilton action within generalized operator $A$ is

$$
S_{A}\left[q_{A}(\cdot)\right]=\int_{a}^{b}\left[p_{A i} \cdot A_{M}^{\alpha} q_{A i}-H_{A}\left(t, q_{A}, p_{A}\right)\right] \mathrm{d} t .
$$

Then,

$$
\delta S_{A}=0
$$

with the commutative conditions [38].

$$
\delta A_{M}^{\alpha} q_{A i}=A_{M}^{\alpha} \delta q_{A i}, \quad i=1,2, \cdots, n
$$

and the boundary conditions

$$
q_{A}(a)=q_{A a}, q_{A}(b)=q_{A b}
$$

is called Hamilton principle within generalized operator $A$, where $\delta$ means the isochronous variation.

Using Equations (1), (8), and (12), we have

$$
\begin{aligned}
\delta S_{A}= & \int_{a}^{b}\left(\delta p_{A i} \cdot A_{M}^{\alpha} q_{A i}+p_{A i} \cdot \delta A_{M}^{\alpha} q_{A i}-\frac{\partial H_{A}}{\partial q_{A i}} \delta q_{A i}-\frac{\partial H_{A}}{\partial p_{A i}} \delta p_{A i}\right) \mathrm{d} t \\
= & \int_{a}^{b}\left[-\left(B_{M^{*}}^{\alpha} p_{A i}+\frac{\partial H_{A}}{\partial q_{A i}}-m p_{A i}(b) \kappa_{1-\alpha}(b, t)+\omega p_{A i}(a) \kappa_{1-\alpha}(t, a)\right)\right. \\
& \left.\cdot \delta q_{A i}+\left(A_{M}^{\alpha} q_{A i}-\frac{\partial H_{A}}{\partial p_{A i}}\right) \delta p_{A i}\right] \mathrm{d} t=0 .
\end{aligned}
$$

Then, from the Hamiltonian $H_{A}=p_{A i} \cdot A_{M}^{\alpha} q_{A i}-L_{A}$, the independence of $\delta q_{A i}$, and the arbitrariness of the interval $[a, b]$, we get

$$
\begin{aligned}
A_{M}^{\alpha} q_{A i}= & \frac{\partial H_{A}}{\partial p_{A i}}, B_{M^{*}}^{\alpha} p_{A i}=-\frac{\partial H_{A}}{\partial q_{A i}}+m p_{A i}(b) \kappa_{1-\alpha}(b, t) \\
& -\omega p_{A i}(a) \kappa_{1-\alpha}(t, a) .
\end{aligned}
$$

Equation (15) is called the Hamilton equation within generalized operator $A$.

Remark 2. Let $\kappa_{\alpha}(t, \tau)=(t-\tau)^{\alpha-1} / \Gamma(\alpha)$; from Equation (15), we can get the Hamilton equations in terms of the left Riemann-Liouville fractional operator, the right RiemannLiouville fractional operator, and the Riesz-RiemannLiouville fractional operator by letting $M=M_{1}, M=M_{2}$, and $M=M_{3}$, respectively.

3.2. Hamilton Equation within Generalized Operator B. Let $L_{B}=L_{B}\left(t, q_{B}, B_{M}^{\alpha} q_{B}\right)$ be the Lagrangian within the generalized operator $B, q_{B}=\left(q_{B 1}, q_{B 2}, \cdots, q_{B n}\right)$, and $B_{M}^{\alpha} q_{B}=\left(B_{M}^{\alpha} q_{B 1}\right.$, $\left.B_{M}^{\alpha} q_{B 2}, \cdots, B_{M}^{\alpha} q_{B n}\right)$, then the generalized momentum $p_{B}=$ $\left(p_{B 1}, p_{B 2}, \cdots, p_{B n}\right)$ is defined as $p_{B i}=\partial L_{B} / \partial B_{M}^{\alpha} q_{B i}, i=1,2, \cdots n$, and the Hamiltonian $H_{B}=H_{B}\left(t, q_{B}, p_{B}\right)$ can be expressed as $H_{B}=p_{B i} \cdot B_{M}^{\alpha} q_{B i}-L_{B}$. Therefore, the Hamilton action within the generalized operator $B$ is

$$
S_{B}\left[q_{B}(\cdot)\right]=\int_{a}^{b}\left[p_{B i} \cdot B_{M}^{\alpha} q_{B i}-H_{B}\left(t, q_{B}, p_{B}\right)\right] \mathrm{d} t
$$

Then,

$$
\delta S_{B}=0
$$


with the commutative conditions [38]

$$
\delta B_{M}^{\alpha} q_{B i}=B_{M}^{\alpha} \delta q_{B i}
$$

and the boundary conditions

$$
q_{B}(a)=q_{B a}, q_{B}(b)=q_{B b}
$$

are called the Hamilton principle within generalized operator $B$, where $\delta$ means the isochronous variation.

Using Equations (9), (17), and (19), we have

$$
\begin{aligned}
\delta S_{B} & =\int_{a}^{b}\left(\delta p_{B i} \cdot B_{M}^{\alpha} q_{B i}+p_{B i} \cdot \delta B_{M}^{\alpha} q_{B i}-\frac{\partial H_{B}}{\partial q_{B i}} \delta q_{B i}-\frac{\partial H_{B}}{\partial p_{B i}} \delta p_{B i}\right) \mathrm{d} t \\
& =\int_{a}^{b}\left[\left(B_{M}^{\alpha} q_{B i}-\frac{\partial H_{B}}{\partial p_{B i}}\right) \delta p_{B i}-\left(A_{M^{*}}^{\alpha} p_{B i}+\frac{\partial H_{B}}{\partial q_{B i}}\right) \delta q_{B i}\right] \mathrm{d} t .
\end{aligned}
$$

Then, from the Hamiltonian $H_{B}=p_{B i} \cdot B_{M}^{\alpha} q_{B i}-L_{B}$, the independence of $\delta q_{B i}$, and the arbitrariness of the interval $[a, b]$, we get

$$
B_{M}^{\alpha} q_{B i}=\frac{\partial H_{B}}{\partial p_{B i}}, A_{M^{*}}^{\alpha} p_{B i}=-\frac{\partial H_{B}}{\partial q_{B i}} .
$$

Equation (21) is called the Hamilton equation within generalized operator $B$.

Remark 3. Equation (21) is consistent with the result obtained in Ref. [39]. However, the method in Ref. [39] is different.

Remark 4. Let $\kappa_{\alpha}(t, \tau)=(t-\tau)^{\alpha-1} / \Gamma(\alpha)$; from Equation (21), we can get the Hamilton equations in terms of the left Caputo fractional operator, the right Caputo fractional operator, and the Riesz-Caputo fractional operator by letting $M=M_{1}, M=M_{2}$, and $M=M_{3}$, respectively.

Remark 5. In Remark 2 and Remark 4, there are six cases in total. If we let $\alpha \longrightarrow 1$, then all of them reduce to the classical Hamilton equation, which can be found in Ref. [14].

\section{Noether Theorems within Generalized Operators}

Noether symmetry means the invariance of the Hamilton action. Noether symmetry leads to conserved quantity. Therefore, the change of the Hamilton action under the infinitesimal transformations will be studied. There are also two parts: one is the Noether symmetry and conserved quantity in terms of operator $A$, and the other is in terms of operator $B$. We begin with the definition of conserved quantity.

Definition 6. A quantity $I$ is called a conserved quantity if and only if the condition $\mathrm{d} I / \mathrm{d} t=0$ holds.
4.1. Noether Theorem within Generalized Operator A. First, we give the infinitesimal transformations within generalized operator $A$ as

$$
\begin{gathered}
\bar{t}=t+\Delta t, \bar{q}_{A i}(\bar{t})=q_{A i}(t)+\Delta q_{A i}, \bar{p}_{A i}(\bar{t})=p_{A i}(t)+\Delta p_{A i}, \\
i=1,2, \cdots, n\left(\text { or } \bar{t}=t+\Delta t, \bar{q}_{A}(\bar{t})=q_{A}(t)+\Delta q_{A}, \bar{p}_{A}(\bar{t})\right. \\
\left.=p_{A}(t)+\Delta p_{A}\right) .
\end{gathered}
$$

Expanding Equation (22), we have

$$
\begin{aligned}
\bar{t} & =t+\theta_{A} \xi_{A 0}^{0}\left(t, q_{A}, p_{A}\right)+o\left(\theta_{A}\right), \bar{q}_{A i}(\bar{t}) \\
& =q_{A i}(t)+\theta_{A} \xi_{A i}^{0}\left(t, q_{A}, p_{A}\right)+o\left(\theta_{A}\right), \bar{p}_{A i}(\bar{t}) \\
& =p_{A i}(t)+\theta_{A} \eta_{A i}^{0}\left(t, q_{A}, p_{A}\right)+o\left(\theta_{A}\right), \quad i=1,2, \cdots, n,
\end{aligned}
$$

where $\theta_{A}$ is an infinitesimal parameter and $\xi_{A 0}^{0}, \xi_{A i}^{0}$, and $\eta_{A i}^{0}$ are called infinitesimal generators within the generalized operator $A$.

Then, let $\Delta S_{A}$ be the linear part of $\bar{S}_{A}-S_{A}$ and neglecting the higher order of $\theta_{A}$, we get

$$
\begin{aligned}
\Delta S_{A}= & \int_{\bar{a}}^{\bar{b}}\left[\bar{p}_{A i} \cdot A_{\bar{M}}^{\alpha} \bar{q}_{A i}-H_{A}\left(\bar{t}, \bar{q}_{A}, \bar{p}_{A}\right)\right] \mathrm{d} \bar{t} \\
& -\int_{a}^{b}\left[p_{A i} \cdot A_{M}^{\alpha} q_{A i}-H_{A}\left(t, q_{A}, p_{A}\right)\right] \mathrm{d} t \\
= & \int_{a}^{b}\left\{( p _ { A i } + \Delta p _ { A i } ) \cdot \left[A_{M}^{\alpha} q_{A i}+A_{M}^{\alpha} \delta q_{A i}+\Delta t \cdot \frac{\mathrm{d}}{\mathrm{d} t} A_{M}^{\alpha} q_{A i}\right.\right. \\
& \left.+\omega \Delta b q_{A i}(b) \frac{\mathrm{d}}{\mathrm{d} t} \kappa_{1-\alpha}(b, t)-m \Delta a q_{A i}(a) \frac{\mathrm{d}}{\mathrm{d} t} \kappa_{1-\alpha}(t, a)\right] \\
& -H_{A}\left(t+\Delta t, q_{A}+\Delta q_{A}, p_{A}+\Delta p_{A}\right)-p_{A i} A_{M}^{\alpha} q_{A i} \\
& \left.+H_{A}\left(t, q_{A}, p_{A}\right)\right\} \mathrm{d} t=\theta_{A} \int_{a}^{b}\left[p_{A i} \cdot A_{M}^{\alpha}\left(\xi_{A i}^{0}-\dot{q}_{A i} \xi_{A 0}^{0}\right)\right. \\
& +\left(p_{A i} \cdot \frac{\mathrm{d}}{\mathrm{d} t} A_{M}^{\alpha} q_{A i}-\frac{\partial H_{A}}{\partial t}\right) \xi_{A 0}^{0}+\left(p_{A i} \cdot A_{M}^{\alpha} q_{A i}-H_{A}\right) \dot{\xi}_{A 0}^{0} \\
& -\frac{\partial H_{A}}{\partial q_{A i}} \xi_{A i}^{0}+\omega p_{A i} \cdot q_{A i}(b) \cdot \xi_{A 0}^{0}\left(b, q_{A}(b), p_{A}(b)\right) \\
& \cdot \frac{\mathrm{d}}{\mathrm{d} t} \kappa_{1-\alpha}(b, t)-m p_{A i} \cdot q_{A i}(a) \cdot \xi_{A 0}^{0}\left(a, q_{A}(a), p_{A}(a)\right) \\
& \left.\cdot \frac{\mathrm{d}}{\mathrm{d} t} \kappa_{1-\alpha}(t, a)\right] \mathrm{d} t,
\end{aligned}
$$

where

$$
\begin{aligned}
& A_{\bar{M}}^{\alpha} \bar{q}_{A i}= A_{M}^{\alpha} q_{A i}+A_{M}^{\alpha} \delta q_{A i}+\Delta t \cdot \frac{\mathrm{d}}{\mathrm{d} t} A_{M}^{\alpha} q_{A i}+\omega q_{A i}(b) \cdot \Delta b \\
& \cdot \frac{\mathrm{d}}{\mathrm{d} t} \kappa_{1-\alpha}(b, t)-m q_{A i}(a) \cdot \Delta a \cdot \frac{\mathrm{d}}{\mathrm{d} t} \kappa_{1-\alpha}(t, a) \\
& \bar{M}=\langle\bar{a}, \bar{t}, \bar{b}, m, \omega>.
\end{aligned}
$$


It follows from Noether symmetry $\left(\Delta S_{A}=0\right)$ that

$$
\begin{aligned}
p_{A i} \cdot A_{M}^{\alpha}\left(\xi_{A i}^{0}-\dot{q}_{A i} \xi_{A 0}^{0}\right)+\left(p_{A i} \cdot \frac{\mathrm{d}}{\mathrm{d} t} A_{M}^{\alpha} q_{A i}-\frac{\partial H_{A}}{\partial t}\right) \xi_{A 0}^{0} \\
+\left(p_{A i} \cdot A_{M}^{\alpha} q_{A i}-H_{A}\right) \dot{\xi}_{A 0}^{0}-\frac{\partial H_{A}}{\partial q_{A i}} \xi_{A i}^{0}+\omega p_{A i} \cdot q_{A i}(b) \\
\cdot \xi_{A 0}^{0}\left(b, q_{A}(b), p_{A}(b)\right) \cdot \frac{\mathrm{d}}{\mathrm{d} t} \kappa_{1-\alpha}(b, t)-m p_{A i} \cdot q_{A i}(a) \\
\cdot \xi_{A 0}^{0}\left(a, q_{A}(a), p_{A}(a)\right) \cdot \frac{\mathrm{d}}{\mathrm{d} t} \kappa_{1-\alpha}(t, a)=0, \quad i=1,2, \cdots, n .
\end{aligned}
$$

Equation (26) is called the Noether identity within the generalized operator $A$.

Finally, the conserved quantities within generalized operators deduced by the Noether symmetry are presented.

Theorem 7. For the Hamiltonian system within generalized operator A (Equation (15)), if the infinitesimal generators $\xi_{A 0}^{0}, \quad \xi_{A i}^{0}$, and $\eta_{A i}^{0}$ satisfy the Noether identity (Equation (26)), then there exists a conserved quantity

$$
\begin{aligned}
I_{A 0}= & \left(p_{A i} \cdot A_{M}^{\alpha} q_{A i}-H_{A}\right) \xi_{A 0}^{0}+\int_{a}^{t}\left\{p_{A i} \cdot A_{M}^{\alpha}\left(\xi_{A i}^{0}-\dot{q}_{A i} \xi_{A 0}^{0}\right)\right. \\
& +\left(\xi_{A i}^{0}-\dot{q}_{A i} \xi_{A 0}^{0}\right) \cdot\left[B_{M^{*}}^{\alpha} p_{A i}-m p_{A i}(b) \kappa_{1-\alpha}(b, \tau)\right. \\
& \left.\left.+\omega p_{A i}(a) \kappa_{1-\alpha}(\tau, a)\right]\right\} d \tau+\omega \cdot q_{A i}(b) \\
& \cdot \xi_{A 0}^{0}\left(b, q_{A}(b), p_{A}(b)\right) \int_{a}^{t} p_{A i}(\tau) \cdot \frac{d}{d \tau} \kappa_{1-\alpha}(b, \tau) d \tau \\
& -m \cdot q_{A i}(a) \cdot \xi_{A 0}^{0}\left(a, q_{A}(a), p_{A}(a)\right) \int_{a}^{t} p_{A i}(\tau) \\
& \cdot \frac{d}{d \tau} \kappa_{1-\alpha}(\tau, a) d \tau=\text { const. }
\end{aligned}
$$

Proof. From Equations (15) and (26), we have $\mathrm{d} I_{A 0} / \mathrm{d} t=0$.

If we let $\Delta S_{A}=-\int_{a}^{b}(\mathrm{~d} / \mathrm{d} t)\left(\Delta G_{A}^{0}\right) \mathrm{d} t, \Delta G_{A}^{0}=\theta_{A} G_{A}^{0}\left(t, q_{A}\right.$, $p_{A}$ ), and $G_{A}^{0}$ be called the gauge function, then from Equation (24), we have

$$
\begin{aligned}
p_{A i} \cdot A_{M}^{\alpha}\left(\xi_{A i}^{0}-\dot{q}_{A i} \xi_{A 0}^{0}\right)+\left(p_{A i} \cdot \frac{\mathrm{d}}{\mathrm{d} t} A_{M}^{\alpha} q_{A i}-\frac{\partial H_{A}}{\partial t}\right) \xi_{A 0}^{0} \\
+\left(p_{A i} \cdot A_{M}^{\alpha} q_{A i}-H_{A}\right) \dot{\xi}_{A 0}^{0}-\frac{\partial H_{A}}{\partial q_{A i}} \xi_{A i}^{0}+\omega p_{A i} \cdot q_{A i}(b) \\
\cdot \xi_{A 0}^{0}\left(b, q_{A}(b), p_{A}(b)\right) \cdot \frac{\mathrm{d}}{\mathrm{d} t} \kappa_{1-\alpha}(b, t)-m p_{A i} \cdot q_{A i}(a) \\
\cdot \xi_{A 0}^{0}\left(a, q_{A}(a), p_{A}(a)\right) \cdot \frac{\mathrm{d}}{\mathrm{d} t} \kappa_{1-\alpha}(t, a)+\dot{G}_{A}^{0}=0, \quad i=1,2, \cdots, n .
\end{aligned}
$$

Equation (28) is called the Noether quasi-identity within the generalized operator $A$.

In this case, we have the following theorem.
Theorem 8. For the Hamiltonian system within the generalized operator A (Equation (15)), if there exists a gauge function $G_{A}^{0}$ such that the infinitesimal generators $\xi_{A 0}^{0}, \xi_{A i}^{0}$, and $\eta_{A i}^{0}$ satisfy the Noether quasi-identity (Equation (28)), then there exists a conserved quantity

$$
\begin{aligned}
I_{A G 0}= & \left(p_{A i} \cdot A_{M}^{\alpha} q_{A i}-H_{A}\right) \xi_{A 0}^{0}+\int_{a}^{t}\left\{p_{A i} \cdot A_{M}^{\alpha}\left(\xi_{A i}^{0}-\dot{q}_{A i} \xi_{A 0}^{0}\right)\right. \\
& +\left(\xi_{A i}^{0}-\dot{q}_{A i} \xi_{A 0}^{0}\right) \cdot\left[B_{M^{*}}^{\alpha} p_{A i}-m p_{A i}(b) \kappa_{1-\alpha}(b, \tau)\right. \\
& \left.\left.+\omega p_{A i}(a) \kappa_{1-\alpha}(\tau, a)\right]\right\} d \tau+\omega \cdot q_{A i}(b) \\
& \cdot \xi_{A 0}^{0}\left(b, q_{A}(b), p_{A}(b)\right) \int_{a}^{t} p_{A i}(\tau) \cdot \frac{d}{d \tau} \kappa_{1-\alpha}(b, \tau) d \tau \\
& +G_{A}^{0}-m \cdot q_{A i}(a) \cdot \xi_{A 0}^{0}\left(a, q_{A}(a), p_{A}(a)\right) \int_{a}^{t} p_{A i}(\tau) \\
& \cdot \frac{d}{d \tau} \kappa_{1-\alpha}(\tau, a) d \tau=\text { const. }
\end{aligned}
$$

Proof. From Equations (15) and (28), we have $\mathrm{d} I_{A \mathrm{G} 0} / \mathrm{d} t=0$.

Remark 9. Let $\kappa_{\alpha}(t, \tau)=(t-\tau)^{\alpha-1} / \Gamma(\alpha)$; from Equations (26) and (28), Theorem 7, and Theorem 8, we can get Noether identities, Noether quasi-identities, and Noether theorems in terms of the left Riemann-Liouville fractional operator, the right Riemann-Liouville fractional operator, and the Riesz-Riemann-Liouville fractional operator by letting $M=$ $M_{1}, M=M_{2}$, and $M=M_{3}$, respectively.

4.2. Noether Theorem within Generalized Operator B. The infinitesimal transformations are

$$
\begin{aligned}
\bar{t}= & t+\Delta t, \bar{q}_{B i}(\bar{t})=q_{B i}(t)+\Delta q_{B i}, \bar{p}_{B i}(\bar{t})=p_{B i}(t)+\Delta p_{B i} \\
& \cdot\left(\operatorname{or} \bar{t}=t+\Delta t, \bar{q}_{B}(\bar{t})=q_{B}(t)+\Delta q_{B}, \bar{p}_{B}(\bar{t})=p_{B}(t)+\Delta p_{B}\right),
\end{aligned}
$$

and the expanded forms of the infinitesimal transformations are

$$
\begin{aligned}
\bar{t}= & t+\theta_{B} \xi_{B 0}^{0}\left(t, q_{B}, p_{B}\right)+o\left(\theta_{B}\right), \bar{q}_{B i}(\bar{t})=q_{B i}(t)+\theta_{B} \xi_{B i}^{0}\left(t, q_{B}, p_{B}\right) \\
& +o\left(\theta_{B}\right), \bar{p}_{B i}(\bar{t})=p_{B i}(t)+\theta_{B} \eta_{B i}^{0}\left(t, q_{B}, p_{B}\right)+o\left(\theta_{B}\right),
\end{aligned}
$$

where $\theta_{B}$ is an infinitesimal parameter and $\xi_{B 0}^{0}, \xi_{B i}^{0}$, and $\eta_{B i}^{0}$ are called infinitesimal generators within the generalized operator $B$. 
Similarly, let $\Delta S_{B}$ be the linear part of $\bar{S}_{B}-S_{B}$ and neglecting the higher order of $\theta_{B}$, we get

$$
\begin{aligned}
\Delta S_{B}= & \bar{S}_{B}-S_{B}=\int_{\bar{a}}^{\bar{b}}\left[\bar{p}_{B i} \cdot B_{\bar{M}}^{\alpha} \bar{q}_{B i}-H_{B}\left(\bar{t}, \bar{q}_{B}, \bar{p}_{B}\right)\right] \mathrm{d} \bar{t} \\
& -\int_{a}^{b}\left[p_{B i} \cdot B_{M}^{\alpha} q_{B i}-H_{B}\left(t, q_{B}, p_{B}\right)\right] \mathrm{d} t \\
= & \int_{a}^{b}\left\{( p _ { B i } + \Delta p _ { B i } ) \cdot \left[B_{M}^{\alpha} q_{B i}+B_{M}^{\alpha} \delta q_{B i}+\Delta t \cdot \frac{\mathrm{d}}{\mathrm{d} t} B_{M}^{\alpha} q_{B i}\right.\right. \\
& \left.+\omega \Delta b \kappa_{1-\alpha}(b, t) \dot{q}_{B i}(b)-m \Delta a \cdot \kappa_{1-\alpha}(t, a) \dot{q}_{B i}(a)\right] \\
& -H_{B}\left(t+\Delta t, q_{B}+\Delta q_{B}, p_{B}+\Delta p_{B}\right)-p_{B i} B_{M}^{\alpha} q_{B i} \\
& \left.+H_{B}\left(t, q_{B}, p_{B}\right)\right\} \mathrm{d} t=\theta_{B} \int_{a}^{b}\left[p_{B i} \cdot B_{M}^{\alpha}\left(\xi_{B i}^{0}-\dot{q}_{B i} \xi_{B 0}^{0}\right)\right. \\
& +\left(p_{B i} \cdot \frac{\mathrm{d}}{\mathrm{d} t} B_{M}^{\alpha} q_{B i}-\frac{\partial H_{B}}{\partial t}\right) \xi_{B 0}^{0}+\left(p_{B i} \cdot B_{M}^{\alpha} q_{B i}-H_{B}\right) \dot{\xi}_{B 0}^{0} \\
& -\frac{\partial H_{B}}{\partial q_{B i}} \xi_{B i}^{0}+\omega \kappa_{1-\alpha}(b, t) \dot{q}_{B i}(b) \cdot p_{B i} \xi_{B 0}^{0}\left(b, q_{B}(b), p_{B}(b)\right) \\
& \left.-m \kappa_{1-\alpha}(t, a) \dot{q}_{B i}(a) p_{B i} \xi_{B 0}^{0}\left(a, q_{B}(a), p_{B}(a)\right)\right] \mathrm{d} t .
\end{aligned}
$$

where

$$
\begin{aligned}
B_{\bar{M}}^{\alpha} \bar{q}_{B i}= & B_{M}^{\alpha} q_{B i}+B_{M}^{\alpha} \delta q_{B i}+\Delta t \cdot \frac{\mathrm{d}}{\mathrm{d} t} B_{M}^{\alpha} q_{B i} \\
& +\omega \kappa_{1-\alpha}(b, t) \dot{q}_{B i}(b) \Delta b-m \kappa_{1-\alpha}(t, a) \dot{q}_{B i}(a) \Delta a .
\end{aligned}
$$

Letting $\Delta S_{B}=0$, we have

$$
\begin{aligned}
p_{B i} \cdot B_{M}^{\alpha}\left(\xi_{B i}^{0}-\dot{q}_{B i} \xi_{B 0}^{0}\right)+\left(p_{B i} \cdot \frac{\mathrm{d}}{\mathrm{d} t} B_{M}^{\alpha} q_{B i}-\frac{\partial H_{B}}{\partial t}\right) \xi_{B 0}^{0} \\
+\left(p_{B i} \cdot B_{M}^{\alpha} q_{B i}-H_{B}\right) \dot{\xi}_{B 0}^{0}-\frac{\partial H_{B}}{\partial q_{B i}} \xi_{B i}^{0}+\omega \kappa_{1-\alpha}(b, t) \dot{q}_{B i}(b) \\
\quad \cdot p_{B i} \xi_{B 0}^{0}\left(b, q_{B}(b), p_{B}(b)\right)-m \kappa_{1-\alpha}(t, a) \dot{q}_{B i} \\
\quad \cdot(a) p_{B i} \xi_{B 0}^{0}\left(a, q_{B}(a), p_{B}(a)\right)=0, \quad i=1,2, \cdots, n
\end{aligned}
$$

Equation (34) is called the Noether identity within the generalized operator $B$.

Let $\Delta S_{B}=-\int_{a}^{b}(\mathrm{~d} / \mathrm{d} t)\left(\Delta G_{B}^{0}\right) \mathrm{d} t, \Delta G_{B}^{0}=\theta_{B} G_{B}^{0}\left(t, \mathbf{q}_{\mathbf{B}}, \mathbf{p}_{\mathbf{B}}\right)$, and $G_{B}^{0}$ be called the gauge function, then from Equation (32), we have

$$
\begin{aligned}
p_{B i} \cdot & B_{M}^{\alpha}\left(\xi_{B i}^{0}-\dot{q}_{B i} \xi_{B 0}^{0}\right)+\left(p_{B i} \cdot \frac{\mathrm{d}}{\mathrm{d} t} B_{M}^{\alpha} q_{B i}-\frac{\partial H_{B}}{\partial t}\right) \xi_{B 0}^{0} \\
& +\left(p_{B i} \cdot B_{M}^{\alpha} q_{B i}-H_{B}\right) \dot{\xi}_{B 0}^{0}-\frac{\partial H_{B}}{\partial q_{B i}} \xi_{B i}^{0}+\omega \kappa_{1-\alpha}(b, t) \dot{q}_{B i}(b) \\
& \cdot p_{B i} \xi_{B 0}^{0}\left(b, q_{B}(b), p_{B}(b)\right)-m \kappa_{1-\alpha}(t, a) \dot{q}_{B i}(a) \\
& \cdot p_{B i} \xi_{B 0}^{0}\left(a, q_{B}(a), p_{B}(a)\right)+\dot{G}_{B}^{0}=0, \quad i=1,2, \cdots, n .
\end{aligned}
$$

Equation (35) is called the Noether quasi-identity within the generalized operator $B$.

Therefore, we have the following theorem.

Theorem 10. For the Hamiltonian system within the generalized operator $B$ (Equation (21)), if the infinitesimal generators $\xi_{B 0}^{0}, \xi_{B i}^{0}$, and $\eta_{B i}^{0}$ satisfy the Noether identity (Equation (34)), then there exists a conserved quantity

$$
\begin{aligned}
I_{B 0}= & \left(p_{B i} \cdot B_{M}^{\alpha} q_{B i}-H_{B}\right) \xi_{B 0}^{0}+\int_{a}^{t}\left[p_{B i} \cdot B_{M}^{\alpha}\left(\xi_{B i}^{0}-\dot{q}_{B i} \xi_{B 0}^{0}\right)\right. \\
& \left.+\left(\xi_{B i}^{0}-\dot{q}_{B i} \xi_{B 0}^{0}\right) A_{M^{*}}^{\alpha} p_{B i}\right] d \tau+\omega \cdot \dot{q}_{B i}(b) \\
& \cdot \xi_{B 0}^{0}\left(b, q_{B}(b), p_{B}(b)\right) \int_{a}^{t} p_{B i}(\tau) \kappa_{1-\alpha}(b, \tau) d \tau-m \cdot \dot{q}_{B i}(a) \\
& \cdot \xi_{B 0}^{0}\left(a, q_{B}(a), p_{B}(a)\right) \int_{a}^{t} p_{B i}(\tau) \kappa_{1-\alpha}(\tau, a) d \tau=\text { const. }
\end{aligned}
$$

Proof. From Equations (21) and (34), we have $\mathrm{d} I_{B 0} / \mathrm{d} t=0$.

Theorem 11. For the Hamiltonian system within the generalized operator B (Equation (21)), if there exists a gauge function $G_{B}^{0}$ such that the infinitesimal generators $\xi_{B 0}^{0}, \xi_{B i}^{0}$, and $\eta_{B i}^{0}$ satisfy the Noether quasi-identity (Equation (35)), then there exists a conserved quantity

$$
\begin{aligned}
I_{B G 0}= & \left(p_{B i} \cdot B_{M}^{\alpha} q_{B i}-H_{B}\right) \xi_{B 0}^{0}+\int_{a}^{t}\left[p_{B i} \cdot B_{M}^{\alpha}\left(\xi_{B i}^{0}-\dot{q}_{B i} \xi_{B 0}^{0}\right)\right. \\
& \left.+\left(\xi_{B i}^{0}-\dot{q}_{B i} \xi_{B 0}^{0}\right) A_{M^{*}}^{\alpha} p_{B i}\right] d \tau+\omega \cdot \dot{q}_{B i}(b) \\
& \cdot \xi_{B 0}^{0}\left(b, q_{B}(b), p_{B}(b)\right) \int_{a}^{t} p_{B i}(\tau) \kappa_{1-\alpha}(b, \tau) d \tau-m \cdot \dot{q}_{B i}(a) \\
& \cdot \xi_{B 0}^{0}\left(a, q_{B}(a), p_{B}(a)\right) \int_{a}^{t} p_{B i}(\tau) \kappa_{1-\alpha}(\tau, a) d \tau+G_{B}^{0}=\text { const. }
\end{aligned}
$$

Proof. From Equations (21) and (35), we have $\mathrm{d} I_{B G 0} / \mathrm{d} t=0$.

Remark 12. Let $\kappa_{\alpha}(t, \tau)=(t-\tau)^{\alpha-1} / \Gamma(\alpha)$; from Equations (34) and (35), Theorem 10, and Theorem 11, we can get Noether identities, Noether quasi-identities, and Noether theorems in terms of the left Caputo fractional operator, the right Caputo fractional operator, and the Riesz-Caputo fractional operator by letting $M=M_{1}, M=M_{2}$, and $M=$ $M_{3}$, respectively. Particularly, the results obtained when $M$ $=M_{3}$ are consistent with the results presented in Ref. [33].

Remark 13. In Remark 9 and Remark 12, there are six cases in total. If we let $\alpha \longrightarrow 1$, then all of them reduce to the classical Noether identity, the classical Noether quasi-identity, and the classical Noether theorem, which can be found in Ref. [14]. 


\section{Adiabatic Invariants within Generalized Operators}

This section begins with the definition of the adiabatic invariant.

Definition 14. A quantity $I_{z}$ is called an adiabatic invariant if $I_{z}$ contains a parameter $\varepsilon$, whose highest power is $z$, and also satisfies that $\mathrm{d} I_{z} / \mathrm{d} t$ is in proportion to $\varepsilon^{z+1}$.

When the systems (Equations (15) and (21)) are disturbed by small forces, the conserved quantities may also change.

Assuming that the Hamiltonian system within the generalized operator $A$ (Equation (15)), the gauge function $G_{A}$ and the infinitesimal generators $\xi_{A 0}, \xi_{A i}$, and $\eta_{A i}$ are disturbed as

$$
\begin{aligned}
B_{M^{*}}^{\alpha} p_{A i}= & -\frac{\partial H_{A}}{\partial q_{A i}}+m p_{A i}(b) \kappa_{1-\alpha}(b, t)-\omega p_{A i}(a) \kappa_{1-\alpha}(t, a) \\
& -\varepsilon_{A} W_{A i}\left(t, q_{A}, p_{A}\right), A_{M}^{\alpha} q_{A i}=\frac{\partial H_{A}}{\partial p_{A i}}, \quad i=1,2, \cdots, n,
\end{aligned}
$$

$$
\begin{aligned}
G_{A}= & G_{A}^{0}+\varepsilon_{A} G_{A}^{1}+\varepsilon_{A}^{2} G_{A}^{2}+\cdots=\varepsilon_{A}^{s} G_{A}^{s}, \xi_{A 0}=\xi_{A 0}^{0}+\varepsilon_{A} \xi_{A 0}^{1} \\
& +\varepsilon_{A}^{2} \xi_{A 0}^{2}+\cdots=\varepsilon_{A}^{s} \xi_{A 0}^{s}, \xi_{A i}=\xi_{A i}^{0}+\varepsilon_{A} \xi_{A i}^{1}+\varepsilon_{A}^{2} \xi_{A i}^{2}+\cdots \\
= & \varepsilon_{A}^{s} \xi_{A i}^{s}, \eta_{A i}=\eta_{A i}^{0}+\varepsilon_{A} \eta_{A i}^{1}+\varepsilon_{A}^{2} \eta_{A i}^{2}+\cdots=\varepsilon_{A}^{s} \eta_{A i}^{s}, \quad s=0,1,2, \cdots
\end{aligned}
$$

then we have the following theorem.

Theorem 15. For the disturbed Hamiltonian system within the generalized operator A (Equation (38)), if there exists a gauge function $G_{A}^{s}$ such that the infinitesimal generators $\xi_{A 0}^{s}, \xi_{A i}^{s}$, and $\eta_{A i}^{s}$ satisfy

$$
\begin{aligned}
p_{A i} \cdot A_{M}^{\alpha}\left(\xi_{A i}^{s}-\dot{q}_{A i} \xi_{A 0}^{s}\right)+\left(p_{A i} \cdot \frac{d}{d t} A_{M}^{\alpha} q_{A i}-\frac{\partial H_{A}}{\partial t}\right) \xi_{A 0}^{s} \\
+\left(p_{A i} \cdot A_{M}^{\alpha} q_{A i}-H_{A}\right) \dot{\xi}_{A 0}^{s}-\frac{\partial H_{A}}{\partial q_{A i}} \xi_{A i}^{s}+\omega p_{A i} \cdot q_{A i}(b) \\
\cdot \xi_{A 0}^{s}\left(b, q_{A}(b), p_{A}(b)\right) \cdot \frac{d}{d t} \kappa_{1-\alpha}(b, t) \\
-W_{A i}\left(\xi_{A i}^{s-1}-\dot{q}_{A i} \xi_{A 0}^{s-1}\right)-m p_{A i} \cdot q_{A i}(a) \\
\cdot \xi_{A 0}^{s}\left(a, q_{A}(a), p_{A}(a)\right) \cdot \frac{d}{d t} \kappa_{1-\alpha}(t, a)+\dot{G}_{A}^{s}=0
\end{aligned}
$$

where $\xi_{A i}^{s-1}=\xi_{A 0}^{s-1}=0$ when $s=0$, then there exists an adiabatic invariant

$$
\begin{aligned}
I_{A G z}= & \sum_{s=0}^{z} \varepsilon_{A}^{s}\left\{\left(p_{A i} \cdot A_{M}^{\alpha} q_{A i}-H_{A}\right) \xi_{A 0}^{s}+\int_{a}^{t}\left\{p_{A i}\right.\right. \\
& \cdot A_{M}^{\alpha}\left(\xi_{A i}^{s}-\dot{q}_{A i} \xi_{A 0}^{s}\right)+\left(\xi_{A i}^{s}-\dot{q}_{A i} \xi_{A 0}^{s}\right) \\
& \left.\cdot\left[B_{M^{*}}^{\alpha} p_{A i}-m p_{A i}(b) \kappa_{1-\alpha}(b, \tau)+\omega p_{A i}(a) \kappa_{1-\alpha}(\tau, a)\right]\right\} d \tau \\
& +\omega \cdot q_{A i}(b) \cdot \xi_{A 0}^{s}\left(b, q_{A}(b), p_{A}(b)\right) \int_{a}^{t} p_{A i}(\tau) \\
& \cdot \frac{d}{d \tau} \kappa_{1-\alpha}(b, \tau) d \tau+G_{A}^{s}-m \cdot q_{A i}(a) \\
& \left.\cdot \xi_{A 0}^{s}\left(a, q_{A}(a), p_{A}(a)\right) \int_{a}^{t} p_{A i}(\tau) \cdot \frac{d}{d \tau} \kappa_{1-\alpha}(\tau, a) d \tau\right\} .
\end{aligned}
$$

Proof. From Equations (38) and (40), we have (d/dt) $I_{A \mathrm{Gz}}$ $=-\varepsilon_{A}^{z+1} W_{A i}\left(\xi_{A i}^{z}-\dot{q}_{A i} \xi_{A 0}^{z}\right)$.

If the Hamiltonian system within the generalized operator $B$ (Equation (21)), the gauge function $G_{B}$ and the infinitesimal generators $\xi_{B 0}, \xi_{B i}$, and $\eta_{B i}$ are disturbed as

$$
\begin{aligned}
& B_{M}^{\alpha} q_{B i}=\frac{\partial H_{B}}{\partial p_{B i}}, A_{M^{*}}^{\alpha} p_{B i} \\
&=-\frac{\partial H_{B}}{\partial q_{B i}}-\varepsilon_{B} W_{B i}\left(t, q_{B}, p_{B}\right), \quad i=1,2, \cdots, n, \\
& G_{B}=G_{B}^{0}+\varepsilon_{B} G_{B}^{1}+\varepsilon_{B}^{2} G_{B}^{2}+\cdots=\varepsilon_{B}^{s} G_{B}^{s}, \xi_{B 0}=\xi_{B 0}^{0}+\varepsilon_{B} \xi_{B 0}^{1} \\
&+\varepsilon_{B}^{2} \xi_{B 0}^{2}+\cdots=\varepsilon_{B}^{s} \xi_{B 0}^{s}, \\
& \xi_{B i}=\xi_{B i}^{0}+\varepsilon_{B} \xi_{B i}^{1}+\varepsilon_{B}^{2} \xi_{B i}^{2}+\cdots=\varepsilon_{B}^{s} \xi_{B i}^{s}, \eta_{B i}=\eta_{B i}^{0}+\varepsilon_{B} \eta_{B i}^{1} \\
&+\varepsilon_{B}^{2} \eta_{B i}^{2}+\cdots=\varepsilon_{B}^{s} \eta_{B i}^{s}, \\
& s=0,1,2, \cdots,
\end{aligned}
$$

then we have the following theorem.

Theorem 16. For the disturbed Hamiltonian system within the generalized operator B (Equation (42)), if there exists a gauge function $G_{B}^{s}$ such that the infinitesimal generators $\xi_{B O}^{s}$, $\xi_{B i}^{s}$, and $\eta_{B i}^{s}$ satisfy

$$
\begin{aligned}
p_{B i} \cdot B_{M}^{\alpha}\left(\xi_{B i}^{s}-\dot{q}_{B i} \xi_{B 0}^{s}\right)+\left(p_{B i} \cdot \frac{d}{d t} B_{M}^{\alpha} q_{B i}-\frac{\partial H_{B}}{\partial t}\right) \xi_{B 0}^{s} \\
+\left(p_{B i} \cdot B_{M}^{\alpha} q_{B i}-H_{B}\right) \dot{\xi}_{B 0}^{s}-\frac{\partial H_{B}}{\partial q_{B i}} \xi_{B i}^{s}+\omega \kappa_{1-\alpha}(b, t) \dot{q}_{B i}(b) \\
\quad \cdot p_{B i} \xi_{B 0}^{s}\left(b, q_{B}(b), p_{B}(b)\right)-W_{B i}\left(\xi_{B i}^{s-1}-\dot{q}_{B i} \xi_{B 0}^{s-1}\right) \\
\quad-m \kappa_{1-\alpha}(t, a) \dot{q}_{B i}(a) p_{B i} \xi_{B 0}^{s}\left(a, q_{B}(a), p_{B}(a)\right)+\dot{G}_{B}^{s}=0
\end{aligned}
$$

where $\xi_{B i}^{s-1}=\xi_{B 0}^{s-1}=0$ when $s=0$, then there exists an adiabatic invariant 


$$
\begin{aligned}
I_{B G z}= & \sum_{s=0}^{z} \varepsilon_{B}^{s}\left\{\left(p_{B i} \cdot B_{M}^{\alpha} q_{B i}-H_{B}\right) \xi_{B 0}^{s}+\int_{a}^{t}\left[p_{B i} \cdot B_{M}^{\alpha}\left(\xi_{B i}^{s}-\dot{q}_{B i} \xi_{B 0}^{s}\right)\right.\right. \\
& \left.+\left(\xi_{B i}^{s}-\dot{q}_{B i} \xi_{B 0}^{s}\right) \cdot A_{M^{*}}^{\alpha} p_{B i}\right] d \tau+\omega \cdot \dot{q}_{B i}(b) \\
& \cdot \xi_{B 0}^{s}\left(b, q_{B}(b), p_{B}(b)\right) \int_{a}^{t} p_{B i}(\tau) \kappa_{1-\alpha}(b, \tau) d \tau-m \cdot \dot{q}_{B i}(a) \\
& \left.\cdot \xi_{B 0}^{s}\left(a, q_{B}(a), p_{B}(a)\right) \int_{a}^{t} p_{B i}(\tau) \kappa_{1-\alpha}(\tau, a) d \tau+G_{B}^{s}\right\} .
\end{aligned}
$$

Proof. From Equations (42) and (46), we have $(\mathrm{d} / \mathrm{d} t) I_{\mathrm{BGz}}=$ $-\varepsilon_{B}^{z+1} W_{B i}\left(\xi_{B i}^{z}-\dot{q}_{B i} \xi_{B 0}^{z}\right)$.

Remark 17. Letting $\kappa_{\alpha}(t, \tau)=(t-\tau)^{\alpha-1} / \Gamma(\alpha)$, we can get the adiabatic invariants in terms of the left Riemann-Liouville fractional operator, the right Riemann-Liouville fractional operator, and the Riesz-Riemann-Liouville fractional operator from Theorem 15 by letting $M=M_{1}, M=M_{2}$, and $M=M_{3}$, respectively. Similarly, we can get the adiabatic invariants in terms of the left Caputo fractional operator, the right Caputo fractional operator, and the Riesz-Caputo fractional operator by letting $M=M_{1}, M=M_{2}$, and $M=$ $M_{3}$, respectively. Particularly, the adiabatic invariant within the Riesz-Caputo fractional operator is consistent with the result obtained in Ref. [33].

Remark 18. In Remark 17, there are six cases in total. If we let $\alpha \longrightarrow 1$, then all of them reduce to the classical adiabatic invariant, which can be found in Ref. [33].

Remark 19. The adiabatic invariants of Theorem 15 and Theorem 16 reduce to conserved quantities of Theorem 8 and Theorem 11 when $z=0$, respectively.

In the following text, we give two applications to illustrate the results and methods.

\section{Applications}

6.1. Application 1. We try to find the conserved quantity and adiabatic invariant for the two-dimensional isotropic harmonic oscillator within the generalized operator $A$, whose Lagrangian has the form

$$
L_{A}=\frac{1}{2} \alpha_{A}\left[\left(A_{M}^{\alpha} q_{A 1}\right)^{2}+\left(A_{M}^{\alpha} q_{A 2}\right)^{2}\right]-\frac{1}{2} \beta_{A}\left[\left(q_{A 1}\right)^{2}+\left(q_{A 2}\right)^{2}\right]
$$

where $\alpha_{A}$ and $\beta_{A}$ are constants.

From Equation (48), we have

$$
\begin{aligned}
p_{A 1} & =\alpha_{A} A_{M}^{\alpha} q_{A 1}, p_{A 2}=\alpha_{A} A_{M}^{\alpha} q_{A 2}, H_{A} \\
& =\frac{p_{A 1}^{2}}{2 \alpha_{A}}+\frac{p_{A 2}^{2}}{2 \alpha_{A}}+\frac{1}{2} \beta_{A}\left[\left(q_{A 1}\right)^{2}+\left(q_{A 2}\right)^{2}\right] .
\end{aligned}
$$

Then, Equation (15) gives

$$
\begin{aligned}
A_{M}^{\alpha} q_{A 1}= & \frac{p_{A 1}}{\alpha_{A}}, A_{M}^{\alpha} q_{A 2}=\frac{p_{A 2}}{\alpha_{A}}, B_{M^{*}}^{\alpha} p_{A 1}=-\beta_{A} q_{A 1} \\
& +m p_{A 1}(b) \kappa_{1-\alpha}(b, t)-\omega p_{A 1}(a) \kappa_{1-\alpha}(t, a), B_{M^{*}}^{\alpha} p_{A 2} \\
= & -\beta_{A} q_{A 2}+m p_{A 2}(b) \kappa_{1-\alpha}(b, t)-\omega p_{A 2}(a) \kappa_{1-\alpha}(t, a) .
\end{aligned}
$$

And under the condition $(\mathrm{d} / \mathrm{d} t) \kappa(t, \tau)=-(\mathrm{d} / \mathrm{d} \tau) \kappa(t, \tau)$, we can verify that

$$
\xi_{A 0}^{0}=1, \xi_{A 1}^{0}=\xi_{A 2}^{0}=0, G_{A}^{0}=0,
$$

satisfy the Noether quasi-identity (Equation (28)). Therefore, from Theorem 8 , we have

$$
\begin{aligned}
I_{A G 0}= & p_{A 1} \cdot A_{M}^{\alpha} q_{A 1}+p_{A 2} \cdot A_{M}^{\alpha} q_{A 2}-H_{A}-\int_{a}^{t}\left\{p_{A 1} \cdot \frac{\mathrm{d}}{\mathrm{d} \tau} A_{M}^{\alpha} q_{A 1}\right. \\
& +\dot{q}_{A 1} \cdot\left[B_{M^{*}}^{\alpha} p_{A 1}-m p_{A 1}(b) \kappa_{1-\alpha}(b, \tau)\right. \\
& \left.+\omega p_{A 1}(a) \kappa_{1-\alpha}(\tau, a)\right]+p_{A 2} \cdot \frac{\mathrm{d}}{\mathrm{d} \tau} A_{M}^{\alpha} q_{A 2} \\
& +\dot{q}_{A 2}\left[B_{M^{*}}^{\alpha} p_{A 2}-m p_{A 2}(b) \kappa_{1-\alpha}(b, \tau)\right. \\
& \left.\left.+\omega p_{A 2}(a) \kappa_{1-\alpha}(\tau, a)\right]\right\} \mathrm{d} \tau=\text { const. }
\end{aligned}
$$

When the system is disturbed by $-\varepsilon_{A} W_{A 1}\left(t, q_{A}, p_{A}\right)=$ $-\varepsilon_{A} q_{A 2}$ and $-\varepsilon_{A} W_{A 2}\left(t, q_{A}, p_{A}\right)=-\varepsilon_{A} q_{A 1}$, then we can find that

$$
\xi_{A 0}^{1}=1, \xi_{A 1}^{1}=\xi_{A 2}^{1}=0, G_{A}^{1}=-q_{A 1} q_{A 2},
$$

is a solution to Equation (40). Therefore, from Theorem 15 , we obtain

$$
I_{A G 1}=I_{A G 0}+\varepsilon_{A}\left(I_{A G 0}-q_{A 1} q_{A 2}\right) .
$$

Specially, let $\kappa_{\alpha}(t, \tau)=(t-\tau)^{\alpha-1} / \Gamma(\alpha)$ and $M=M_{1}$ (or $M=M_{2}$ or $M=M_{3}$ ); further, letting $\alpha \longrightarrow 1$, we have

$$
I_{A G 0 C}=-H_{A}=\text { const, } I_{A G 1 C}=-H_{A}-\varepsilon_{A}\left(H_{A}+q_{A 1} q_{A 2}\right) \text {. }
$$

6.2. Application 2. The Lotka biochemical oscillator model's Hamiltonian has the form

$$
H_{B}=\alpha_{B 1} p_{B 1}-\alpha_{B 2} q_{B 1}+\beta_{B 1} \exp p_{B 1}-\beta_{B 2} \exp q_{B 1} .
$$

We try to find its conserved quantity and adiabatic invariant based on generalized operator $B$. 
Equation (21) gives the Hamilton equation within the generalized operator $B$

$$
\begin{aligned}
B_{M}^{\alpha} q_{B 1} & =\alpha_{B 1}+\beta_{B 1} \exp p_{B 1}, B_{M}^{\alpha} q_{B 2}=0, A_{M^{*}}^{\alpha} p_{B 1} \\
& =\alpha_{B 2}+\beta_{B 2} \exp q_{B 1}, A_{M^{*}}^{\alpha} p_{B 2}=0 .
\end{aligned}
$$

Then, under the condition $(\mathrm{d} / \mathrm{d} t) \kappa(t, \tau)=-(\mathrm{d} / \mathrm{d} \tau) \kappa(t, \tau)$, it is easy to verify that

$$
\xi_{B 0}^{0}=1, \xi_{B 1}^{0}=0, G_{B}^{0}=0
$$

is a solution to the Noether quasi-identity (Equation (35)). Therefore, from Theorem 11, we have

$$
\begin{aligned}
I_{B G 0}= & p_{B 1} \cdot B_{M}^{\alpha} q_{B 1}-H_{B} \\
& -\int_{a}^{t}\left(\dot{q}_{B 1} A_{M^{*}}^{\alpha} p_{B 1}+p_{B 1} \cdot \frac{\mathrm{d}}{\mathrm{d} \tau} B_{M}^{\alpha} q_{B 1}\right) \mathrm{d} \tau=\text { const. }
\end{aligned}
$$

When the system is disturbed by $-\varepsilon_{B} W_{B 1}\left(t, q_{B}, p_{B}\right)=$ $-\varepsilon_{B}\left(2 q_{B 1}+1\right)$ and $-\varepsilon_{B} W_{B 2}\left(t, q_{B}, p_{B}\right)=0$, then we can find that

$$
\xi_{B 0}^{1}=1, \xi_{B 1}^{1}=0, G_{B}^{1}=-q_{B 1}^{2}-q_{B 1},
$$

is a solution to Equation (46). Therefore, from Theorem 16 , we have

$$
\begin{aligned}
I_{B G 1}= & p_{B 1} \cdot B_{M}^{\alpha} q_{B 1}-H_{B}-\int_{a}^{t}\left(\dot{q}_{B 1} A_{M^{*}}^{\alpha} p_{B 1}+p_{B 1} \cdot \frac{\mathrm{d}}{\mathrm{d} \tau} B_{M}^{\alpha} q_{B 1}\right) \mathrm{d} \tau \\
& +\varepsilon_{B}\left[p_{B 1} \cdot B_{M}^{\alpha} q_{B 1}-H_{B}-\int_{a}^{t}\left(\dot{q}_{B 1} A_{M^{*}}^{\alpha} p_{B 1}+p_{B 1} \cdot \frac{\mathrm{d}}{\mathrm{d} \tau} B_{M}^{\alpha} q_{B 1}\right)\right. \\
& \left.\cdot \mathrm{d} \tau-q_{B 1}^{2}-q_{B 1}\right] .
\end{aligned}
$$

Specially, let $\kappa_{\alpha}(t, \tau)=(t-\tau)^{\alpha-1} / \Gamma(\alpha)$ and $M=M_{1}$ (or $M=M_{2}$ or $M=M_{3}$ ); further, letting $\alpha \longrightarrow 1$, we have

$$
I_{B G 0 C}=-H_{B}=\text { const, } I_{B G 1 C}=-H_{B}-\varepsilon_{B}\left(H_{B}+q_{B 1}^{2}+q_{B 1}\right) \text {. }
$$

\section{Results and Discussion}

Hamilton equations, Noether theorems, and adiabatic invariants are obtained for the Hamiltonian systems on the basis of the generalized operators. The Hamilton equation in terms of the generalized operator $A$ (Equation (15)), the Hamilton equation in terms of the generalized operator $B$ (Equation (21)), the Noether symmetries and conserved quantities in terms of the generalized operator $A$ (Theorem 7 and Theorem 8), the Noether symmetries and conserved quantities in terms of the generalized operator $B$ (Theorem 10 and Theorem 11), the perturbation to Noether symmetries and adiabatic invariants in terms of the generalized fractional operators (Theorem 15 and Theorem 16) are all new work.

However, we still need to make efforts to finish the follow-up work successfully. For example, MATLAB is a useful tool to simulate the results of the applications to directly illustrate the achievements obtained in this paper. Therefore, the appliance of MATLAB is necessary. Besides, except for the Noether symmetry method, the Lie symmetry method, and the Mei symmetry method are also significant methods to be studied for the Hamiltonian systems within generalized operators. Time scale calculus is recently proposed with tremendous potential for applications. The Hamiltonian mechanics within mixed time scale and generalized operators is a new research direction, which also deserves to be done.

\section{Data Availability}

No data were used to support this study.

\section{Conflicts of Interest}

The authors declare that they have no conflicts of interest.

\section{Authors' Contributions}

Chuan-Jing Song wrote the first draft of the manuscript. All authors reviewed and approved the final manuscript.

\section{Acknowledgments}

This work was supported by the National Natural Science Foundation of China (grant numbers 11802193, 11801396, and 11972241), the Natural Science Foundation of Jiangsu Province (grant numbers BK20191454 and BK20170374), and the Young Scientific and Technological Talents Promotion Project of Suzhou Association for Science and Technology.

\section{References}

[1] Z. X. Zhu, Q. Z. Zhou, and J. S. Yin, Theoretical Mechanics (II), Peking University Press, Beijing, 1982.

[2] Z. D. Huang and F. E. Zhong, Engineering System Analytical Mechanics, Higher Education Press, Beijing, 1992.

[3] B. Chen, Analytical Dynamics, Peking University Press, Beijing, 2012.

[4] F. X. Mei, H. B. Wu, and Y. M. Li, A Brief History of Analytical Mechanics, Science Press, Beijing, 2019.

[5] Y. Y. Zhao, "New integral variational principle of mechanics," Acta Mechanica Sinica, vol. 21, pp. 101-106, 1989.

[6] G. D. Birkhoff, Dynamical Systems, AMS, New York, 1927.

[7] R. M. Santilli, Foundations of Theoretical Mechanics II, Springer-Verlag, New York, 1983.

[8] F. X. Mei, Dynamics of Generalized Birkhoffian Systems, Science Press, Beijing, 2013.

[9] B. Vujanović, "A variational principle for non-conservative dynamical systems," ZAMM, vol. 55, no. 6, pp. 321-331, 1975. 
[10] J. B. Li, X. H. Zhao, and Z. R. Liu, Generalized Hamiltonian System Theory and Its Applications, Science Press, Beijing, 2007.

[11] F. X. Mei, R. C. Shi, and Y. F. Zhang, Dynamics of Birkhoffian System, Beijing Institute of Technology Press, Beijing, 1996.

[12] A. E. Noether, Invariante Variationsprobleme, Nachr Akad Wiss Gött Math-Phys, KI, 1918.

[13] Л. С. Полак, Вариационные Принципь Механики, ГИФМЛ, Москва, 1959.

[14] F. X. Mei, Analytical Mechanics (II), Beijing Institute of Technology Press, Beijing, 2013.

[15] J. Song and Y. Zhang, "Noether's theorems for dynamical systems of two kinds of non-standard Hamiltonians," Acta Mechanica, vol. 229, no. 1, pp. 285-297, 2018.

[16] A. Mouchet, "Applications of Noether conservation theorem to Hamiltonian systems," Annalen der Physik, vol. 372, pp. 260-282, 2016.

[17] X. H. Zhai and Y. Zhang, "Conservation laws for a delayed Hamiltonian system in a time scales version," Symmetry, vol. 10, no. 12, p. 668, 2018.

[18] X. Tian and Y. Zhang, "Noether symmetry and conserved quantity for Hamiltonian system of Herglotz type on time scales," Acta Mechanica, vol. 229, no. 9, pp. 3601-3611, 2018.

[19] Y. Zhang, "Noether theory for Hamiltonian systems on time scales," Chinese Quarterly of Mechanics, vol. 37, pp. 214-224, 2016.

[20] Q. H. Zu and J. Q. Zhu, "Noether theorem for nonholonomic nonconservative mechanical systems in phase space on time scales," Journal of Mathematical Physics, vol. 57, no. 8, article 082701, 2016

[21] F. X. Mei and H. B. Wu, Analytical Mechanics Methods of Differential Equations, Science Press, Beijing, 2012.

[22] I. Podlubny, Fractional Differential Equations, Academic Press, New York, 1999.

[23] R. L. Magin, Fractional Calculus in Bioengineering, Begell House Publisher, Connecticut, 2006.

[24] C. J. Song and O. P. Agrawal, "Hamiltonian formulation of systems described using fractional singular Lagrangian," Acta Applicandae Mathematicae, vol. 172, no. 1, p. 9, 2021.

[25] D. Baleanu and O. P. Agrawal, "Fractional Hamilton formalism within Caputo's derivative," Czechoslovak Journal of Physics, vol. 56, no. 10-11, pp. 1087-1092, 2006.

[26] E. M. Rabei, K. I. Nawafleh, R. S. Hijjawi, S. I. Muslih, and D. Baleanu, "The Hamilton formalism with fractional derivatives," Journal of Mathematical Analysis and Applications, vol. 327, no. 2, pp. 891-897, 2007.

[27] F. Riewe, "Nonconservative Lagrangian and Hamiltonian mechanics," Physical Review E, vol. 53, no. 2, pp. 1890-1899, 1996.

[28] M. Klimek, "Lagrangean and Hamiltonian fractional sequential mechanics," Czechoslovak Journal of Physics, vol. 52, no. 11, pp. 1247-1253, 2002.

[29] M. A. E. Herzallah and D. Baleanu, "Fractional-order EulerLagrange equations and formulation of Hamiltonian equations," Nonlinear Dynamics, vol. 58, no. 1-2, pp. 385-391, 2009.

[30] S. I. Muslih and D. Baleanu, "Hamiltonian formulation of systems with linear velocities within Riemann-Liouville fractional derivatives," Journal of Mathematical Analysis and Applications, vol. 304, no. 2, pp. 599-606, 2005.
[31] T. M. Atanacković, S. Konjik, L. Oparnica, and S. Pilipović, "Generalized Hamilton's principle with fractional derivatives," Journal of Physics A: Mathematical and Theoretical, vol. 43, no. 25, article 255203, 2010.

[32] T. M. Atanacković and S. Pilipović, "Hamilton's principle with variable order fractional derivatives," Fractional Calculus and Applied Analysis, vol. 14, no. 1, pp. 94-109, 2011.

[33] C. J. Song, "Noether symmetry for fractional Hamiltonian system," Physics Letters A, vol. 383, no. 29, article 125914, 2019.

[34] C. J. Song and Y. Cheng, "Conserved quantity and adiabatic invariant for Hamiltonian system with variable order," Symmetry, vol. 11, no. 10, p. 1270, 2019.

[35] X. Tian and Y. Zhang, "Fractional time-scales Noether theorem with Caputo $\Delta$ derivatives for Hamiltonian systems," Applied Mathematics and Computation, vol. 393, article 125753, 2021.

[36] S. Zhou, H. Fu, and J. L. Fu, "Symmetry theories of Hamiltonian systems with fractional derivatives," Science China - Physics Mechanics \& Astronomy, vol. 54, no. 10, pp. 1847-1853, 2011.

[37] O. P. Agrawal, "Generalized variational problems and EulerLagrange equations," Computers \& Mathematcs with Applications, vol. 59, no. 5, pp. 1852-1864, 2010.

[38] H. B. Zhang and H. B. Chen, "Generalized variational problems and Birkhoff equations," Nonlinear Dynamics, vol. 83, no. 1-2, pp. 347-354, 2016.

[39] C. J. Song, "Generalized fractional forced Birkhoff equations," Journal of Dynamics and Control, vol. 17, pp. 446-452, 2019. 